\title{
LM-STEM Study of Dislocations in Thick Silicon
}

\author{
D. Tang, A. Rucki*, H. Cerva*, and P. Schlossmacher \\ FEI Company, Achtseweg Noord 5, 5600 KA Eindhoven, Netherlands \\ *Siemens AG, CT MM7, Otto-Hahn-Ring 6, 81739 München, Germany
}

It is well known that resolution is one of the key specifications of TEM and STEM applications. For imaging, however, the need for a large field-of-view (FOV) is frequently overlooked. There are cases in which features have to be investigated using a FOV as large as possible. Recently we studied dislocations in thick Si wafer using LMSTEM (LowMag-STEM) technique which provides both visibility of the defects and a very large FOV.

During manufacturing of integrated silicon devices doping of well defined surface regions is often done by ion implantation. This process generally induces extended dislocation structures within the implantation affected surface zone which need to be cured by subsequent high temperature annealing. Even few remaining dislocations may drastically increase the leakage current of the electrical device. TEM investigations allow more precise localization and characterization of these dislocations, but evidently have to be performed on samples cross-sections which are several micrometers thick. High transmission at a large FOV opens the field for 3D defect investigations in silicon semiconductors [1]

Due to higher transmission TEM investigations on thick samples are preferentially performed at $300 \mathrm{kV}$ or $400 \mathrm{kV}$ accelerating voltage. For thick samples conventional TEM images are strongly affected by chromatic aberrations of the imaging lenses due to inelastic scattering of the electrons within the specimen. Resolution of STEM images is not limited in the same way and is thus favorable for TEM imaging on thick samples [2]. TEM samples from an integrated silicon device were cut using a FIB (see SEM image in Fig.1). The four thin windows are different in thicknesses which were estimated to range from $0.96 \mu \mathrm{m}$ to $3.6 \mu \mathrm{m}$. The sample was observed on a Tecnai F30 ST operated at $300 \mathrm{kV}$. A HAADF detector was used to record the STEM images.

At high magnification, dislocations within the device surface zone can be detected, but the FOV is limited. A few dislocations can be clearly observed within the $0.96 \mu \mathrm{m}$ thin window area in TEM mode (Fig. 2; MAG on CCD $=11,5 \mathrm{kx}$ ) as well as in HM-STEM mode (Fig.3; MAG $=12 \mathrm{kx}$ ), but in both cases the FOV amounts only to a few micrometers.

Going to LM-STEM mode, because of very large camera length, STEM is no longer in a DF imaging mode but more in a BF imaging mode since the bright-field disk falls on the HAADF detector. Fig. 4 shows a part of a LM STEM image which was digitally enlarged to enhance the visibility of the same dislocations as in Fig.2; more dislocations within the $0.96 \mu \mathrm{m}$ thin window are also seen. In fact, the original FOV of the image is more than $40 \mu \mathrm{m}$.

Figure 5 is another STEM image taken in LM-STEM mode. The microscope's nominal magnification is 3,400x. All four thin windows are within the FOV. It is also possible to observe dislocations in all those windows. The only challenge is to find the right brightness/contrast settings to see all dislocations, which are in very different specimen thick- 
ness, in a common display. That is the reason why the image of the thinnest window (to the right in Fig. 5) is actually displayed differently from the others (as insert). Fig.5 provides a FOV of about $50 \mu \mathrm{m}$, which can be compared to the SEM image in Fig.1. However, dislocations are only observable in the LM STEM image and not in the SEM image.

\section{References}

[1] Andreas Rucki and Hans Cerva, ECS Trans. 10 (1), 97 (2007)

[2] John M. Cowley, Diffraction Physics, $2^{\text {nd }}$ revised edition, Elsevier 1981

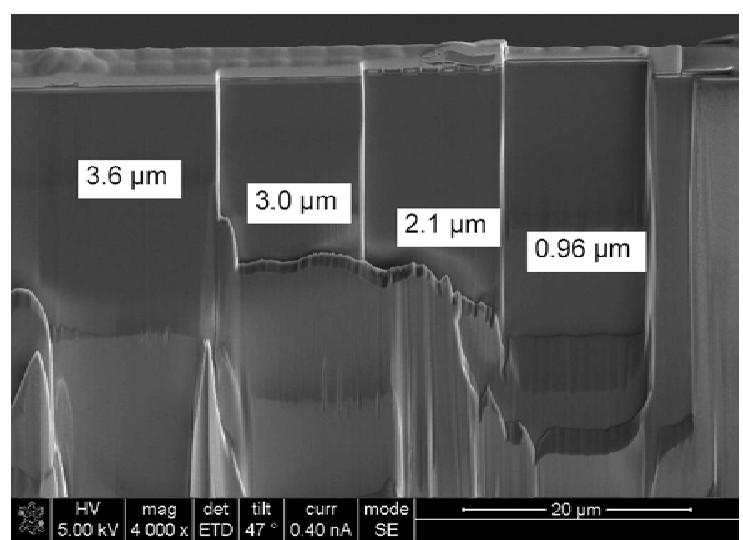

FIG. 1. SEM image of the FIB cut sample with four windows of different thickness.

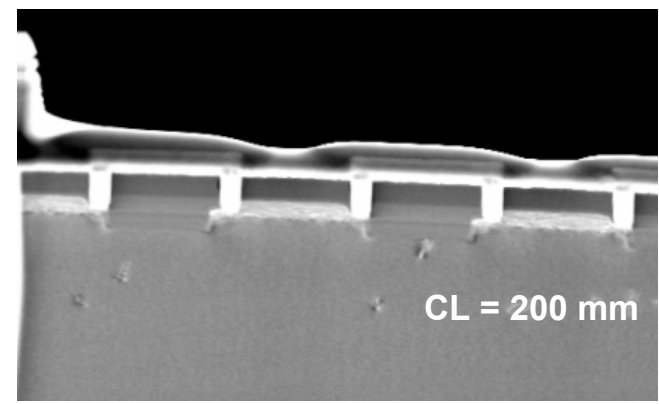

FIG. 3. STEM-HAADF image showing similar dislocations as in Fig. 2. The camera length (CL) was $200 \mathrm{~mm}$. FOV is about $8 \mu \mathrm{m}$.

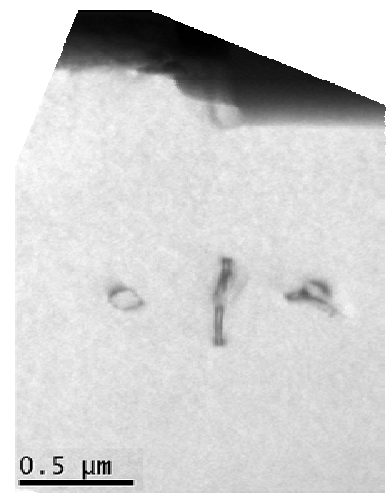

FIG. 2. High-magnification TEM BF image showing a few dislocations. FOV is about $2 \mu \mathrm{m}$.

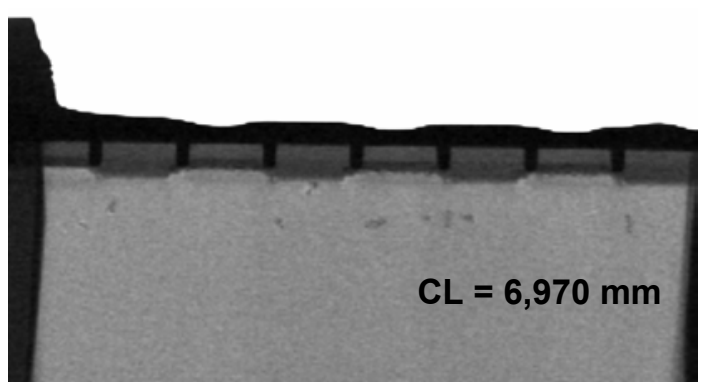

FIG. 4. Digitally enlarged detail of a Low Mag STEM image showing the same dislocations as in Fig. 2 and Fig.3. $\mathrm{CL}=6,970 \mathrm{~mm} ; \mathrm{MAG}=3,400 \mathrm{x} ; \mathrm{FOV} \sim 10 \mu \mathrm{m}$.

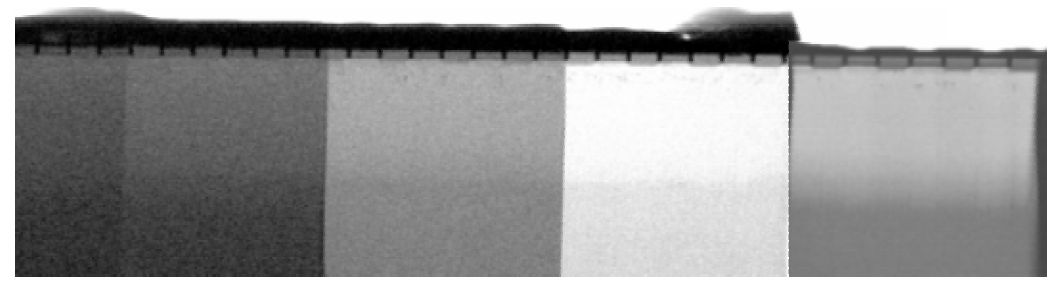

FIG. 5. LM STEM image of which the detail in Fig. 4 was cut out. The nominal magnification is $3,400 \mathrm{x}$. The FOV is $\sim 50 \mu \mathrm{m}$ and thus comparable to the one of Fig. 1, however with discernible dislocation contrast (reproduction/print prevents better visibility). 